\title{
Anti-de Sitter/Conformal-Field-Theory Calculation of Screening in a Hot Wind
}

\author{
Hong Liu, ${ }^{1}$ Krishna Rajagopal, ${ }^{1,2}$ and Urs Achim Wiedemann ${ }^{3}$ \\ ${ }^{1}$ Center for Theoretical Physics, Massachusetts Institute of Technology, Cambridge, Massachusetts 02139, USA \\ ${ }^{2}$ Nuclear Science Division, MS 70R319, Lawrence Berkeley National Laboratory, Berkeley, California 94720, USA \\ ${ }^{3}$ Department of Physics, CERN, Theory Division, CH-1211 Geneva 23, Switzerland
}

(Received 18 July 2006; revised manuscript received 30 October 2006; published 1 May 2007)

\begin{abstract}
One of the challenges in relating experimental measurements of the suppression in the number of $J / \psi$ mesons produced in heavy ion collisions to lattice QCD calculations is that whereas the lattice calculations treat $J / \psi$ mesons at rest, in a heavy ion collision a $c \bar{c}$ pair can have a significant velocity with respect to the hot fluid produced in the collision. The putative $J / \psi$ finds itself in a hot wind. We present the first rigorous nonperturbative calculation of the consequences of a wind velocity $v$ on the screening length $L_{s}$ for a heavy quark-antiquark pair in hot $\mathcal{N}=4$ supersymmetric QCD. We find $L_{s}(v, T)=f(v)\left[1-v^{2}\right]^{1 / 4} / \pi T$ with $f(v)$ only mildly dependent on $v$ and the wind direction. This $L_{s}(v, T) \sim L_{s}(0, T) / \sqrt{\gamma}$ velocity scaling, if realized in QCD, provides a significant additional source of $J / \psi$ suppression at transverse momenta which are high but within experimental reach.
\end{abstract}

Twenty years ago, Matsui and Satz suggested that because the attraction between a quark and an antiquark is screened in a deconfined quark-gluon plasma, the production of $J / \psi$ mesons should be suppressed in sufficiently energetic nucleus-nucleus collisions relative to that in protonproton or proton-nucleus collisions, since the screened interaction between a $c$ and $\bar{c}$ immersed in a quark-gluon plasma would not bind them [1]. In the intervening years, marked progress on many fronts has not changed this basic qualitative picture. On the experimental side, we now have data from the NA50 and NA60 experiments at the CERN SPS and from the PHENIX experiment at RHIC that demonstrate the existence of a suppression [2]. On the theoretical side, we now have ab initio calculations of the temperature-dependent potential between a color-singlet heavy quark and antiquark separated by a distance $L[3,4]$. This potential is as at $T=0$ for small $L$, but begins to weaken for $L$ larger than some $L_{s}$ and flattens at larger $L$. The potentials obtained in these lattice calculations can be crudely characterized as indicating $L_{s} \sim 0.5 / T$ in hot QCD with two flavors of light quarks [4] and $L_{s} \sim 0.7 / T$ in hot QCD with no dynamical quarks [3]. Furthermore, lattice QCD calculations of the Minkowski space $J / \psi$ spectral function itself have now been done in quenched QCD [5], and early results in QCD with dynamical quarks have also been reported [6]. These studies indicate that the $J / \psi$ meson ceases to exist as a bound state above a temperature somewhere between $1.5 T_{c}$ and $2.5 T_{c}$, in agreement with conclusions drawn based upon the screening potential between static quarks [7].

The multifaceted challenge, now, is to make quantitative contact between the lattice calculations and data from heavy ion collisions. One significant difficulty is that the lattice calculations treat a quark-antiquark pair in the quark-gluon plasma rest frame, whereas in a heavy ion collision a $c \bar{c}$ pair is not produced at rest. This challenge becomes more acute in higher energy collisions: at LHC energies, $c \bar{c}$ pairs, which if produced in vacuum would yield $J / \psi$ mesons with transverse momenta many times their rest mass, will be copious. Even in collisions at SPS and RHIC energies, the collective flow developed by the hot medium in which the $c \bar{c}$ pair finds itself is considerable. A rigorous determination of the $v$ dependence of the screening length $L_{s}(T)$ for a heavy quark-antiquark pair in a quark-gluon plasma moving with velocity $v$ would therefore be a significant advance. We provide one, albeit for hot $\mathcal{N}=4$ super Yang-Mills theory.

$\mathcal{N}=4$ super Yang-Mills (SYM) theory is a conformally invariant theory with two parameters: the rank of the gauge group $N_{c}$ and the 't Hooft coupling $\lambda=g_{\mathrm{YM}}^{2} N_{c}$. We shall define the screening length $L_{s}$ below, based upon an analysis of a fundamental Wilson loop describing the dynamics of a color-singlet quark-antiquark "dipole" moving with velocity $v$ along, say, the $x_{3}$ direction through the hot, strongly interacting $\mathcal{N}=4$ SYM plasma. In the rest frame of the dipole, which sees a hot wind blowing in the $x_{3}$ direction, the contour $\mathcal{C}$ of the Wilson loop is given by a rectangle with large extension $\mathcal{T}$ in the $t$ direction, and short sides of length $L$ along some spatial direction. Evaluating this Wilson loop (whether ultimately in QCD or in $\mathcal{N}=4$ SYM theory as we do here) will teach us about the $L$-dependent color-singlet quark-antiquark potential and hence allow us to define a screening length in the presence of a hot wind.

According to the anti-de Sitter/conformal-field-theory (AdS/CFT) correspondence [8], in the large- $N_{c}$ and large- $\lambda$ limits, the thermal expectation value $\left\langle W^{F}(\mathcal{C})\right\rangle$ for the Wilson loop in the absence of a wind velocity can be calculated using the metric for a five-dimensional curved space-time describing a black hole in AdS space [9]. Calling the fifth dimension $r$, the black hole horizon is at some $r=r_{0}$ and we add a probe D3-brane extended along the $x_{1}, x_{2}, x_{3}$ directions at some $r=\Lambda \gg r_{0}$. The external quarks described by the Wilson loop are open strings 
ending on the probe brane. The prescription for evaluating $\left\langle W^{F}(\mathcal{C})\right\rangle$ is that we must find the extremal action surface in the five-dimensional AdS space-time whose boundary at $r=\Lambda$ is the contour $\mathcal{C}$ in Minkowski space $R^{3,1} \cdot\left\langle W^{F}(\mathcal{C})\right\rangle$ is then given by $\exp [i S(\mathcal{C})]=\exp [i E(\mathcal{C}) \mathcal{T}]$, with $S$ the action of the extremal surface [9]. For the timelike Wilson loop we analyze, $S(\mathcal{C})$ is proportional to the time $\mathcal{T}$, meaning that $E(\mathcal{C})$ can be interpreted as the energy of the dipole. In the limit of infinitely heavy quarks, i.e., $\Lambda \rightarrow \infty, E(\mathcal{C}) \propto \Lambda$, but this divergence comes from the $L$-independent selfenergy of the quark or antiquark taken separately. We are only interested in the $L$-dependent part of $E(\mathcal{C})$, which is finite in the $\Lambda \rightarrow \infty$ limit, and we take this limit henceforth [10].

To describe a hot wind in the $x_{3}$ direction, we boost the five-dimensional AdS black hole metric, obtaining

$$
\begin{aligned}
d s^{2}= & -A d t^{2}+2 B d t d x_{3}+C d x_{3}^{2}+r^{2}\left(d x_{1}+d x_{2}^{2}\right) \\
& +f^{-1} d r^{2},
\end{aligned}
$$

where $f=\frac{r^{2}}{R^{2}}\left(1-\frac{r_{0}^{4}}{r^{4}}\right)$ with $R$ the curvature radius of the AdS space and where we have defined

$$
A=\frac{r^{2}}{R^{2}}-\frac{r_{1}^{4}}{r^{2} R^{2}}, \quad B=\frac{r_{1}^{2} r_{2}^{2}}{r^{2} R^{2}}, \quad C=\frac{r^{2}}{R^{2}}+\frac{r_{2}^{4}}{r^{2} R^{2}},
$$

and $r_{1}^{4}=r_{0}^{4} \cosh ^{2} \eta=r_{0}^{4} \gamma^{2}$ and $r_{2}^{4}=r_{0}^{4} \sinh ^{2} \eta=r_{0}^{4} \gamma^{2} v^{2}$, with $v$ the velocity of the wind, $\eta$ its rapidity, and $\gamma=$ $1 / \sqrt{1-v^{2}}$. Here, the temperature $T$ of the Yang-Mills theory at $v=0$ is given by the Hawking temperature of the black hole, $T=\frac{r_{0}}{\pi R^{2}}$, and $R$ and the string tension $1 / 2 \pi \alpha^{\prime}$ are related to the t' Hooft coupling by $\frac{R^{2}}{\alpha^{\prime}}=\sqrt{\lambda}$.

The short side of $\mathcal{C}$ can be chosen to lie in the $\left(x_{1}, x_{3}\right)$ plane, at an angle $\theta \in[0, \pi / 2]$ relative to the $x_{3}$ direction. $\theta$ is the angle between the dipole and the wind direction in the dipole rest frame. We parameterize the surface whose action $S(\mathcal{C})$ is to be extremized by $x^{\mu}=x^{\mu}(\tau, \sigma)$, where $\sigma^{\alpha}=(\tau, \sigma)$ denote world sheet coordinates. The NambuGoto action for the string world sheet is given by

$$
S=\frac{1}{2 \pi \alpha^{\prime}} \int d \sigma d \tau \sqrt{-\operatorname{det} g_{\alpha \beta}}
$$

with $g_{\alpha \beta}=G_{\mu \nu} \partial_{\alpha} x^{\mu} \partial_{\beta} x^{\nu}$ the induced metric on the world sheet. This action is invariant under coordinate changes of $\sigma^{\alpha}$, and we can set $\tau=t$ and $\sigma=x_{1}$ [11]. Note that $\sigma$ takes values $-\frac{L}{2} \sin \theta<\sigma<\frac{L}{2} \sin \theta$. Since $\mathcal{T} \gg L$, we can assume that the surface is translationally invariant along the $\tau$ direction, i.e. $x^{\mu}(\sigma, \tau)=x^{\mu}(\sigma)$. The Wilson loop lies at constant $x_{2}$, so $x_{2}(\sigma)=$ const. For $x_{3}(\sigma)$, one takes $x_{3}\left( \pm \frac{L}{2} \sin \theta\right)= \pm \frac{L}{2} \cos \theta$ as the boundary condition. For the bulk coordinate $r(\sigma)$, we implement the requirement that the world sheet has $\mathcal{C}$ as its boundary by imposing $r\left( \pm \frac{L}{2} \sin \theta\right)=\Lambda \rightarrow \infty$. It proves convenient to define $y \equiv r / r_{0}$ and $z \equiv x_{3} r_{0} / R^{2}=x_{3} \pi T$ and hence $\ell \equiv$ $L r_{0} / R^{2}=L \pi T$ and to define a rescaled $\tilde{\sigma}=\sigma r_{0} / R^{2}$ and promptly drop the tilde. The boundary conditions become

$$
y\left( \pm \frac{\ell}{2} \sin \theta\right)=\infty, \quad z\left( \pm \frac{\ell}{2} \sin \theta\right)= \pm \frac{\ell}{2} \cos \theta .
$$

The action (3) now takes the form

$$
\frac{S}{\mathcal{T}}=E=K \int_{0}^{\frac{l}{2}} d \sigma \mathcal{L},
$$

with the constant prefactor given by $K=\sqrt{\lambda} T$ and with the Lagrangian

$$
\mathcal{L}=\sqrt{\left(y^{4}-\cosh ^{2} \eta\right)\left(1+\frac{y^{\prime 2}}{y^{4}-1}\right)+z^{\prime 2}\left(y^{4}-1\right)},
$$

where henceforth ${ }^{\prime} \equiv \frac{\partial}{\partial \sigma}$. The equations of motion obtained from this Lagrangian can be cast in the form

$$
\begin{gathered}
q^{2} y^{\prime 2}=\left(y^{4}-\cosh ^{2} \eta\right)\left(y^{4}-1-p^{2}\right)-q^{2}\left(y^{4}-1\right), \\
q^{2} z^{\prime 2}=p^{2}\left(\frac{y^{4}-\cosh ^{2} \eta}{y^{4}-1}\right)^{2},
\end{gathered}
$$

where $p$ and $q$ are integration constants. Since $y^{\prime}$ depends only on $y$ and the boundary condition for $y$ is symmetric under $\sigma \leftrightarrow-\sigma$, we find $y(\sigma)$ to be an even function of $\sigma$ : it descends $\left(y^{\prime}<0\right)$ for $-\ell / 2<\sigma<0$, turns around at $\sigma=0$ where $y^{\prime}=0$, and then ascends. Since $z^{\prime}$ depends only on $y$ and the boundary condition for $z$ is antisymmetric in $\sigma \rightarrow-\sigma$, we find $z(\sigma)$ to be an odd function of $\sigma$. In particular, $z=0$ at $\sigma=0$.

Equations (7) and (8) with the boundary conditions (4) can be integrated numerically, and in Fig. 1 we present an example. Somewhat counterintuitively, the projection of the string world sheet onto the $\left(x_{1}, x_{3}\right)$-plane is not a straight line connecting the quark and the antiquark, but rather has a sinusoidal form. This behavior arises for all values of $\theta$ except $\theta=0$ or $\pi / 2$ (wind parallel or perpendicular to the dipole) for which the projection is indeed a straight line. Furthermore, we see that even though there is a wind blowing in the $z$ direction, $y(\sigma)$ is even and $z(\sigma)$ is odd for any angle $\theta$, meaning that the string world sheet is
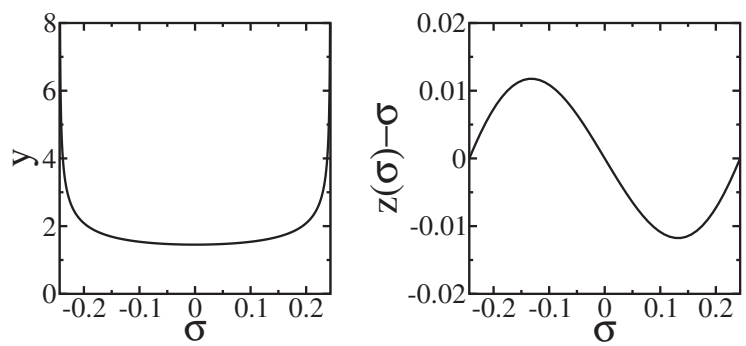

FIG. 1. String world sheet for wind with velocity $v=0.7$ blowing at an angle $\theta=45^{\circ}$ relative to the dipole. The solution has integration constants $p=1.325$ and $q=1.109$, which correspond to $\theta=45^{\circ}$ and $\ell=0.689$. (This $\ell$ is the maximum possible for this $v$ and $\theta$.) $\sigma \propto x_{1}$ extends from $-(\ell / 2) \sin \theta$ to $+(\ell / 2) \sin \theta$. (a) $y(\sigma)$. (b) $z(\sigma)-\sigma$ is the deviation of the string world sheet away from $z=\sigma$, the straight line at $\theta=45^{\circ}$ between the quark and the antiquark. 
not dragged at all by this wind. This conclusion is antithetical to that for the world sheet of an isolated string ending on a single quark or antiquark, analyzed in Ref. [12], and in qualitative agreement with the conclusion that "mesons feel no drag" reached in a different context in Ref. [13]. The $\theta=\pi / 2$ case is particularly simple to analyze because the integration constant $p$ vanishes. With $p=0, z(\sigma)$ is constant, starkly making the point that the world sheet is not dragged by the wind blowing in the $z$ direction.

We shall find that the screening length behaves similarly for all values of $\theta$. For purposes of illustration, we present the analysis for $\theta=\pi / 2$. With $z(\sigma)=$ const, we need only analyze the shape of the world sheet in the $r$ direction, governed by (7) which we can write as $y^{\prime}=\frac{1}{q} \times$ $\sqrt{\left(y^{4}-1\right)\left(y^{4}-y_{c}^{4}\right)}$ upon defining $y_{c}^{4}=\cosh ^{2} \eta+q^{2}$. The world sheet stretches from $y=\infty$ down to $y=y_{c}$, with $y^{\prime}(0)=0$ and $y(0)=y_{c}$. The integration constant $q$ can now be determined from the equation $\frac{\ell}{2}=\int_{0}^{\frac{\ell}{2}} d \sigma$, which becomes

$$
\ell=2 q \int_{y_{c}}^{\infty} d y \frac{1}{\sqrt{\left(y^{4}-y_{c}^{4}\right)\left(y^{4}-1\right)}}
$$

The energy can be written as

$$
E=K \int_{y_{c}}^{\infty} d y \frac{y^{4}-\cosh ^{2} \eta}{\sqrt{\left(y^{4}-1\right)\left(y^{4}-y_{c}^{4}\right)}}
$$

and can be made finite by subtracting the self-energy of an isolated quark and antiquark.

In Fig. 2(a) we plot $\ell$ as a function of $q$ from Eq. (9) for several values of $\cosh ^{2} \eta$. Analysis of Eq. (9) shows that, for any $\cosh ^{2} \eta, \ell$ decreases at large $q$ like $\ell \approx 1.198 / \sqrt{q}$. This corresponds to an energy $E \propto-K \sqrt{q} \propto-K / \ell \propto$ $-\sqrt{\lambda} / L$, and thus describes a quark and antiquark separated by a small distance $L$ interacting via an attractive Coulomb potential. Small values of $L$ can also be achieved by choosing $q \rightarrow 0$, as $\ell \propto q$ at small $q$. These world sheets
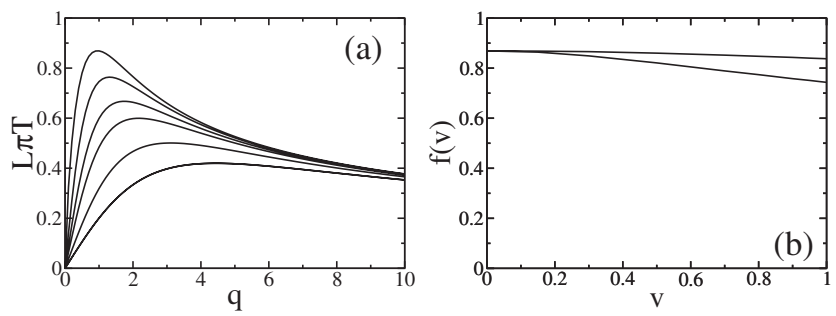

FIG. 2. The dependence of the screening length on the wind velocity $v$. (a) $\ell=L \pi T$, given in Eq. (9), as a function of the integration constant $q$ for six different values of $v: 0,0.5,0.7$, $0.8,0.9,0.95$ (top to bottom). We see the peak of this curve, $\ell_{\max }$, dropping with $v$. All curves are for a wind blowing in the direction perpendicular to the dipole. (b) $f(v)=\ell_{\max } \sqrt{\gamma}=$ $L_{s} \pi T \sqrt{\gamma}$ versus $v$ for a wind blowing perpendicular to the dipole (lower curve) or parallel to the dipole (upper curve). have $E \propto$ const $+L^{2}$, meaning that these small $L$ solutions have much higher energy than those describing Coulomb attraction, and so are not of interest. Since $\ell$ is everywhere positive and goes to zero for both small and large $q$, at some $q_{m}$ it has a maximum value $\ell_{\max }$ as illustrated in Fig. 2(a). For $\ell>\ell_{\max }$, no extremal world sheet bounded by the Wilson loop $\mathcal{C}$ exists, with the exception of disconnected world sheets "hanging from" the quark or the antiquark Wilson line separately, describing their self energies. Hence, for $\ell>\ell_{\max }$ there is no $L$-dependent potential between the quark and antiquark [14]. We can therefore define a screening length $L_{s}$ by $L_{s} \equiv \ell_{\max } / \pi T$. Analysis of (9) shows that for $v=0, q_{m}=0.96$ and $\ell_{\max }=0.869$ are numbers of order 1 while for large $\cosh ^{2} \eta, q_{m} \approx$ $1.42 \cosh \eta$ and $\ell_{\max } \approx 0.743 / \sqrt{\cosh \eta}=0.743\left(1-v^{2}\right)^{1 / 4}$. This motivates writing the screening length as

$$
L_{s}=\frac{f(v)}{\pi T}\left(1-v^{2}\right)^{1 / 4},
$$

in so doing defining the function $f(v)$. We plot $f(v)$ in Fig. 2(b), and find that its velocity dependence is mild meaning that the dominant $v$ dependence of $L_{s}$ is the factor $\left(1-v^{2}\right)^{1 / 4}$. For general $\theta$, the results are very similar: the interaction is screened for $L>L_{s}$ where $L_{s}$ is given by (11) with a slightly different $f(v)$, shown in Fig. 2(b) for a wind blowing parallel to the dipole. As the angle $\theta$ between wind and dipole changes from $0^{\circ}$ to $90^{\circ}, f(v)$ interpolates between the two curves in Fig. 2(b).

Our central result is that, in $\mathcal{N}=4$ SYM theory, the dominant dependence of the screening length of a dipole in a hot wind on the wind velocity is $L_{s}(v, T) \sim L_{s}(0, T) / \sqrt{\gamma}$, with the remaining weak dependence described by the function $f(v)$ in Fig. 2(b). The dominant velocity dependence suggests that $L_{s}$ should be thought of as proportional to (energy density) ${ }^{-1 / 4}$, since the energy density increases like $\gamma^{2}$ as the wind velocity is boosted. It turns out that $L_{s}(0, T)$ is within a factor of 2 of that for QCD. Although it would be interesting to see whether $f(0)$ is closer to that in QCD in more QCD-like theories with gravity duals, given the availability of reliable lattice calculations of the screening potential in QCD itself this is not a pressing issue. It would certainly be interesting to see whether $L_{s}(v, T) \sim$ $L_{s}(0, T) / \sqrt{\gamma}$ persists in other theories with gravity duals, as this would support its applicability to QCD [15].

If the velocity scaling of $L_{s}$ that we have discovered holds for QCD, it will have qualitative consequences for quarkonium suppression in heavy ion collisions. For illustrative purposes, consider the explanation of the $J / \psi$ suppression seen at SPS and RHIC energies proposed in Refs. [7,16]: lattice calculations of the $q \bar{q}$-potential indicate that the $J / \psi(1 S)$ state dissociates at a temperature $\sim 2.1 T_{c}$ whereas the excited $\chi_{c}(2 P)$ and $\psi^{\prime}(2 S)$ states cannot survive above $\sim 1.2 T_{c}$; so, if collisions at both the SPS and RHIC reach temperatures above $1.2 T_{c}$ but not above $2.1 T_{c}$, the experimental facts (comparable anomalous suppression of $J / \psi$ production at the SPS and RHIC) 


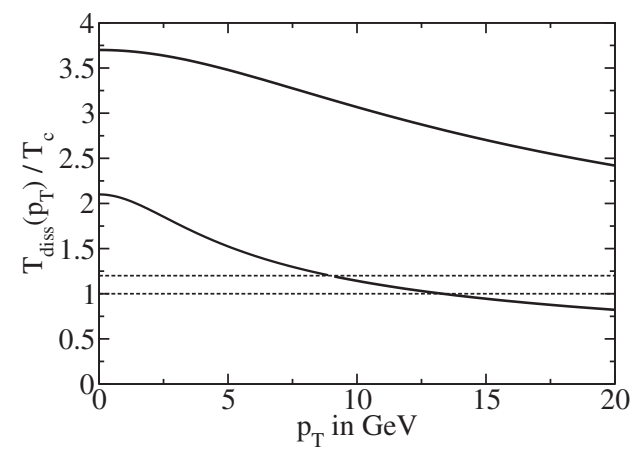

FIG. 3. A $1 / \sqrt{\gamma}$-velocity scaling of the screening length in QCD would imply a $J / \psi$ dissociation temperature $T_{\text {diss }}\left(p_{T}\right)$ that decreases significantly with $p_{T}$, while that for the heavier $Y$ is affected less at a given $p_{T}$. The $Y$ curve is schematic: we have increased $T_{\text {diss }}(0)$ over that for the $J / \psi$ by a factor corresponding to its smaller size in vacuum.

can be understood as the complete loss of the "secondary" $J / \psi$ 's that would have arisen from the decays of the excited states, with no suppression at all of $J / \psi$ 's that originate as $J / \psi$ 's. Taking Eq. (11) at face value, the temperature $T_{\text {diss }}$ needed to dissociate the $J / \psi$ decreases $\propto\left(1-v^{2}\right)^{1 / 4}$. As can be seen from Fig. 3, this indicates that $J / \psi$ suppression at RHIC will increase markedly (as the $J / \psi(1 S)$ mesons themselves dissociate) for $J / \psi$ 's with transverse momentum $p_{T}$ above some threshold that is at most $\sim 9 \mathrm{GeV}$ and would be $\sim 5 \mathrm{GeV}$ if the temperatures reached at RHIC are $\sim 1.5 T_{c}$ [17]. These illustrative considerations point to a novel quarkonium suppression pattern at transverse momenta above $5 \mathrm{GeV}$, a regime that is within experimental reach of future high-luminosity runs at RHIC and that will be studied thoroughly at the LHC. If the temperatures reached at the LHC are, say, $\sim 3 T_{c}$, the LHC could discover $Y$ suppression, but only at high enough $p_{T}$.

H. L. is supported in part by the A.P. Sloan Foundation and the U.S. Department of Energy (DOE) OJI program. Research supported in part by the DOE under cooperative research agreement No. DF-FC02-94ER40818.

[1] T. Matsui and H. Satz, Phys. Lett. B 178, 416 (1986).

[2] B. Alessandro et al. (NA50 Collaboration), Eur. Phys. J. C 39, 335 (2005); R. Arnaldi et al. (NA60 Collaboration), Nucl. Phys. A774, 711 (2006); H. Pereira Da Costa et al. (PHENIX Collaboration), Nucl. Phys. A774, 747 (2006)

[3] O. Kaczmarek, F. Karsch, F. Zantow, and P. Petreczky, Phys. Rev. D 70, 074505 (2004); 72, 059903(E) (2005).

[4] O. Kaczmarek and F. Zantow, Phys. Rev. D 71, 114510 (2005).

[5] M. Asakawa and T. Hatsuda, Phys. Rev. Lett. 92, 012001 (2004); S. Datta, F. Karsch, P. Petreczky, and I. Wetzorke, Phys. Rev. D 69, 094507 (2004).

[6] R. Morrin et al., Proc. Sci. LAT2005 (2005) 176.
[7] F. Karsch, D. Kharzeev, and H. Satz, Phys. Lett. B 637, 75 (2006).

[8] J. M. Maldacena, Adv. Theor. Math. Phys. 2, 231 (1998); E. Witten, Adv. Theor. Math. Phys. 2, 505 (1998); S. S. Gubser, I. R. Klebanov, and A. M. Polyakov, Phys. Lett. B 428, 105 (1998).

[9] J. M. Maldacena, Phys. Rev. Lett. 80, 4859 (1998); S. J. Rey and J. T. Yee, Eur. Phys. J. C 22, 379 (2001); S. J. Rey, S. Theisen, and J. T. Yee, Nucl. Phys. B527, 171 (1998); A. Brandhuber, N. Itzhaki, J. Sonnenschein, and S. Yankielowicz, Phys. Lett. B 434, 36 (1998); J. Sonnenschein, hep-th/0003032.

[10] At this point, the present calculation becomes qualitatively distinct (even in the $v \rightarrow 1$ limit) from the calculation of the lightlike Wilson loop used to determine the "jetquenching parameter" $\hat{q}$ in Refs. [19,20].

[11] With the exception of a wind parallel to the dipole $(\theta=$ $0)$, which can be formulated easily as a special case.

[12] C. P. Herzog, A. Karch, P. Kovtun, C. Kozcaz, and L. G. Yaffe, J. High Energy Phys. 07 (2006) 013; J. CasalderreySolana and D. Teaney, Phys. Rev. D 74, 085012 (2006); S. S. Gubser, Phys. Rev. D 74, 126005 (2006); C.P. Herzog, J. High Energy Phys. 09 (2006) 032; E. Caceres and A. Guijosa, J. High Energy Phys. 11 (2006) 077; J. J. Friess, S. S. Gubser, and G. Michalogiorgakis, J. High Energy Phys. 09 (2006) 072; Y.h. Gao, W.s. Xu, and D.f. Zeng, hep-th/0606266; J. J. Friess, S.S. Gubser, G. Michalogiorgakis, and S. S. Pufu, Phys. Rev. D (to be published).

[13] K. Peeters, J. Sonnenschein, and M. Zamaklar, Phys. Rev. D 74, 106008 (2006). See also Appendix A of first paper in [12].

[14] In this theory, as in QCD, one expects a residual attraction that falls exponentially with $L$ for $L>L_{s}$. Seeing such effects (which are nonperturbative in $\alpha^{\prime}$ ) requires analysis beyond extremizing the Nambu-Goto action.

[15] In a hot wind, the large-spin mesons in the confining, nonsupersymmetric theory studied by Peeters, Sonnenschein, and Zamaklar [13] dissociate beyond a maximum wind velocity. The relation between the size $L$ of these mesons and their dissociation velocity $v$ is consistent with $L \propto$ $\left(1-v^{2}\right)^{1 / 4}$, in qualitative agreement with the result we have obtained analytically in a simpler setting.

[16] S. Digal, P. Petreczky, and H. Satz, Phys. Lett. B 514, 57 (2001); H. Satz, Nucl. Phys. A783, 249 (2007).

[17] Any $J / \psi$ mesons formed by recombination will have transverse momenta much lower than those at which our calculation is relevant. Also, it is only at much higher $p_{T}$ that one has to take into account the possibility that $J / \psi$ mesons could form outside the hot medium [18].

[18] F. Karsch and R. Petronzio, Z. Phys. C 37, 627 (1988).

[19] H. Liu, K. Rajagopal, and U. A. Wiedemann, Phys. Rev. Lett. 97, 182301 (2006).

[20] A. Buchel, Phys. Rev. D 74, 046006 (2006); J. F. VazquezPoritz, hep-th/0605296; E. Caceres and A. Guijosa, J. High Energy Phys., 12 (2006) 068; F. L. Lin and T. Matsuo, Phys. Lett. B 641, 45 (2006); S. D. Avramis and K. Sfetsos, J. High Energy Phys. 01 (2007) 065; N. Armesto, J. D. Edelstein, and J. Mas, J. High Energy Phys. 09 (2006) 039. 